\title{
Design of Fast Transient Response, LOW Dropout REgulator WITH ENHANCED STEADY STATE CHARACTERISTICS ON THE BASIS OF PID CONTROL
}

\author{
Alhassan Mumuni ${ }^{1}$ and Fuseini Mumuni ${ }^{2}$ \\ ${ }^{1}$ Electrical/Electronics Engineering Department, \\ Cape Coast Polytechnic, Cape Coast, Ghana \\ ${ }^{2}$ Electrical and Electronics Engineering Department, \\ University of Mines and Technology, Tarkwa, Ghana
}

\begin{abstract}
Design and implementation of control systems for power supplies require the use of efficient techniques that provide simple and practical solutions in order to fulfill the performance requirements at an acceptable cost. Application of manual methods of system identification in determining optimal values of controller settings is quite time-consuming, expensive and, sometimes, may be impossible to practically carry out. This paper describes an analytical method for the design of a control system for a fast transient response, low dropout (LDO) linear regulated power supply on the basis of PID compensation. The controller parameters are obtained from analytical model of the regulator circuit. Test results showed good dynamic characteristics with adequate margin of stability. This study shows that PID parameter values sufficiently close to optimum can easily be obtained from analytical study of the regulator system. The applied method of determining controller settings greatly reduces design time and cost.
\end{abstract}

\section{KEYWORDS}

Low dropout (LDO) regulator, small-signal analysis, transient response, PID controller tuning

\section{INTRODUCTION}

Most electronic devices use low-voltage DC supplies, which usually obtain energy from mains AC. These power supplies are generally constructed using traditional linear or switching regulator circuits, with a bridge rectifier and simple capacitor filter connected to the network via a transformer. Linear regulators are usually preferred to switching regulators for low power applications because of their extreme simplicity, reliability and low cost [1-2]. Linear regulators typically incorporate a series or shunt pass element in the form of a bipolar or a FET transistor, a control circuit in the form of DC comparator, a voltage reference and a feedback network as shown in Figure 1. 


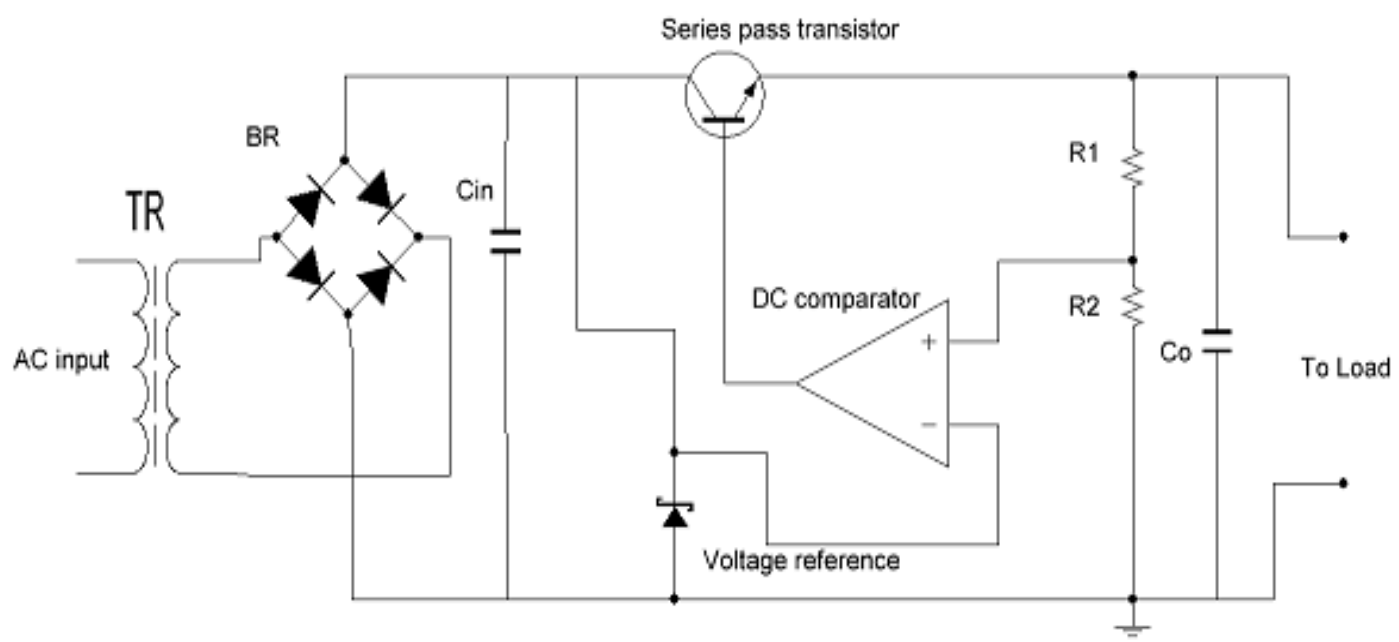

Figure 1. Conventional LDO regulator structure

Using these simple architectures, a practically constant output can be obtained regardless of the current drawn by the load and any minor fluctuations in the input voltage. However, in recent years, the emergence of noise sensitive electronic circuits has made it difficult for traditional linear regulators to satisfy the stringent supply requirements. Maintaining the output within acceptable limits requires advanced control circuitry that is capable of executing sophisticated control algorithms. There are many advanced compensation techniques offering enhanced regulation performance [1]. Often, this is achieved at the expense of simplicity, cost and efficiency. However, a power supply control circuitry needs to be simple, cost-effective and practical to implement. Such a regulator can be realized using Proportional, Integral and Differential (PID) controllers. PID control has gained much prominence in power control applications because of the simplicity of its construction, ease of use, clarity of operation, suitability for most practical problems and low cost. Using such a compensator allows the dynamic characteristics of the regulator to be controlled by adjusting only three parameters, thus making it possible to obtain satisfactory regulation performance with simpler control circuits.

The first step in designing a robust controller for a regulated power supply is the synthesis of an accurate model of the system. There are several techniques for accomplishing this. These techniques are based on experimental studies or theoretical analysis. Characterizing low power electronic components experimentally requires extreme precision, and consequently, sophisticated and expensive laboratory equipment. Analytical methods provide simple and cost effective means of obtaining the dynamic characteristics of the system.

The objective of this paper is to design a high precision linear regulator with fast transient response and zero steady state error by using controller of fixed structure. The manual method of determining controller settings has the disadvantage that it can be very difficult and expensive to identify numerical values for the controller parameters. Therefore, in this study we use an analytical method for producing a mathematical model of the regulator: the dynamic characteristics of the model were determined theoretically and PID controller coefficients calculated according to these characteristics. 


\section{Proposed LDO Regulator Structure AND CONTROL SCHEME}

In published literature, different regulator architectures, which have advantages in specific applications, have been proposed [2-6]. The adequacy of a regulator structure is determined by the requirements of the technical specifications for the power supply. Usually, the main requirements for the supply are maintenance of the set output voltage within the limits permitted by the load, the size, power dissipation and speed of response. The LDO regulator proposed in this study utilizes a P-channel MOSFET (PMOS) device as a pass element because of the superior low dropout voltage and high temperature stability of MOSFET devices. Also, unlike BJT devices, MOSFETs do not have steady-state gate current, leading to higher efficiency. A PID controller is used as the control element for the regulator. A PID controller can be constructed in different ways [7]. For the purpose of this study we use a PID structure of the form shown in Figure 2 whose mathematical model is written as

$$
u(t)=k_{p}\left[e(t)+\frac{1}{\tau_{i}} \int_{0}^{t} e(t) d t+\tau_{d} \frac{e(t)}{d t}\right]
$$

And its transfer function is, thus

$$
\frac{U_{C}(s)}{E(s)}=k_{p}\left(1+\frac{1}{\tau_{i} s}+\tau_{d} s\right)
$$

where $k_{p}$ is the proportional gain, $\tau_{i}=\frac{1}{K_{i}}$ is the integral time constant, and $\tau_{d}=K_{d}$ is the derivative time constant.

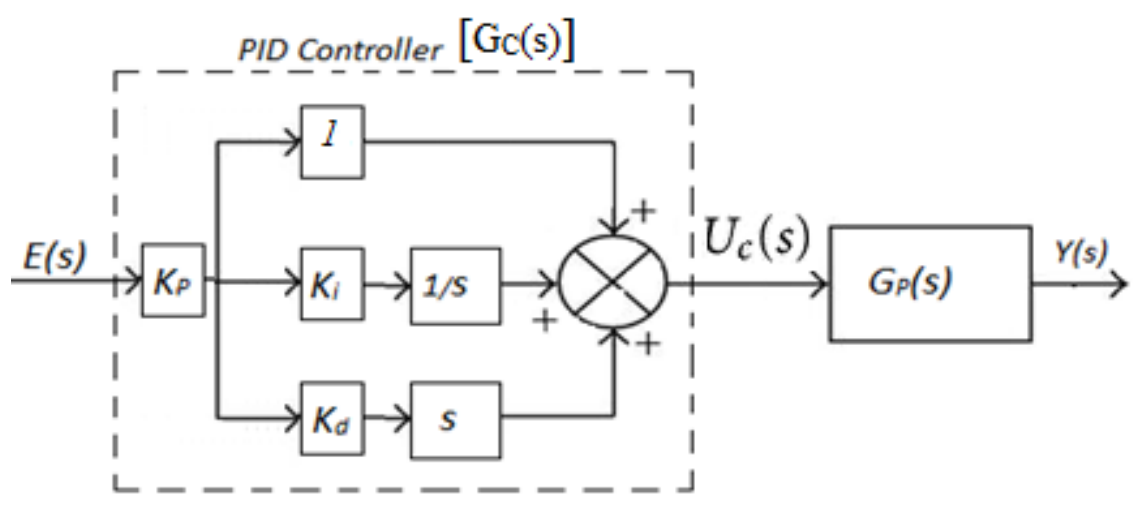

Figure 2. PID regulator structure

A PID controller satisfying the above description can be realized using operational amplifiers. A schematic diagram of the proposed LDO regulator with the PID controller is presented in Figure 3. It features a P-channel MOSFET as the series pass element, a DC amplifier as a comparator, and resistive feed-back network. There is also a filtering stage consisting of an output capacitor $C_{O}$ with an equivalent series resistor $R_{E S R}$ and a bypass capacitor $C_{b}$. These are added to reduce 
International Journal of Computational Science, Information Technology and Control Engineering (IJCSITCE) Vol.3, No.3, July 2016

output ripples and provide stability. The structure of the scheme includes a PID controller whose parameters can be set according the required dynamic characteristics of the power supply system. Since the PID controller has a negative transfer function, an inverting buffer IB is connected to its output to give a resultant positive gain.

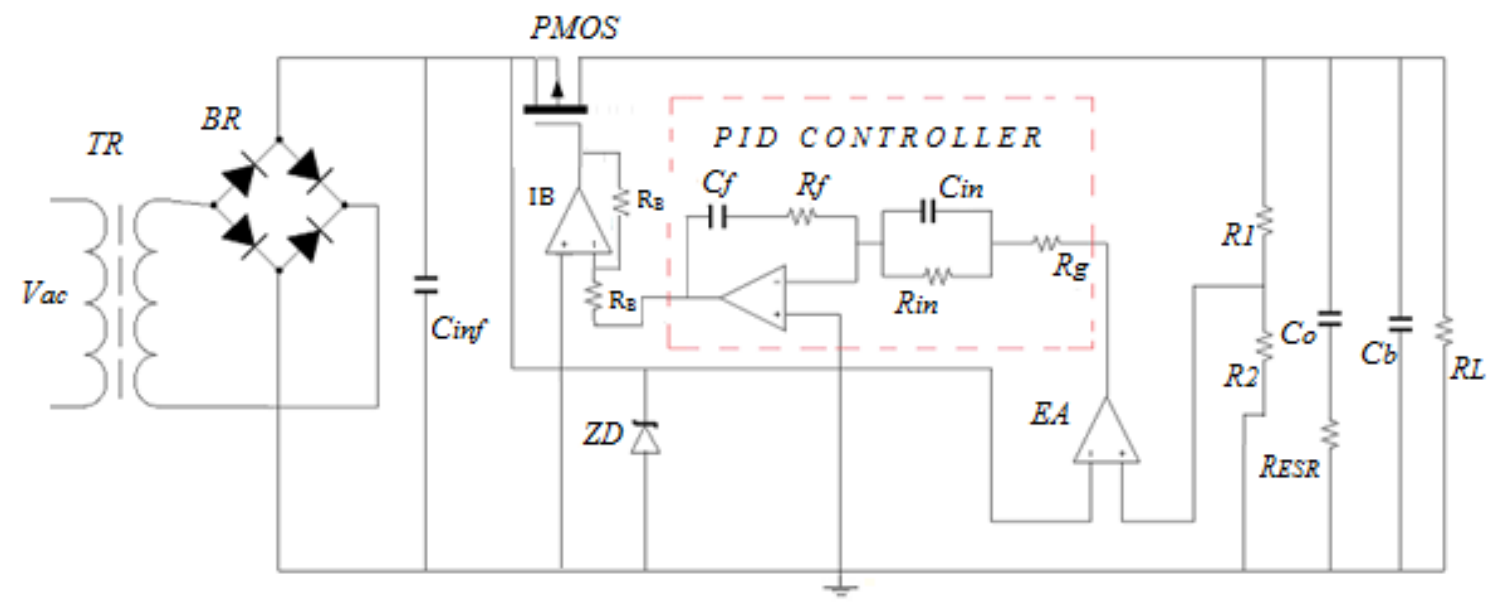

Figure 3. Proposed LDO regulator structure and controller architecture

The proposed controller shown in Figure 3 can be represented in the transfer function form

$$
G_{c}(s)=-\frac{\left(\tau_{1} s+1\right)\left(\tau_{2} s+1\right)}{\tau_{1} s} \frac{1}{1+s \tau_{g}}=-\left[k_{p}\left(1+\frac{\tau_{2}}{\tau_{1}}\right)+\frac{1}{\tau_{i}} s+\tau_{d} s\right] \frac{1}{1+s \tau_{g}}
$$

where

$$
\tau_{1}=R_{f} C_{f}, \tau_{2}=R_{i n} C_{i n}, \tau_{i}=\left(R_{i n}+R_{g}\right) C_{f}, k_{p}=\frac{R_{f}}{R_{i n}+R_{g}}, \tau_{d}=\frac{R_{1} R_{f} C_{i n}}{R_{i n}+R_{g}}, \text { and } \tau_{g}=\frac{R_{i n} R_{g} C_{i n}}{R_{i n}+R_{g}} .
$$

PID parameters $k_{p}, \tau_{i}$ and $\tau_{d}$ can be set by appropriately selecting the values of $C_{i n}, C_{f}, R_{i n}$ and $R_{f} . R_{g}$ is optionally included to introduce a low pass filter $L_{p}(s)=\frac{1}{1+s \tau_{g}}$ to provide a steeper roll-off of the controller gain at high frequencies.

\section{ANALYTICAL MODEL OF THE LOW DROPOUT POWER REgUlator}

Design of a high performance linear regulator requires accurate knowledge of the pass element and the associated components, as well as a complete characterization of the resulting system. This requires a precise calculation of all system parameters. Basic parameters of analog devices are determined by small signal analysis [8], so for their calculation it is necessary to use small signal equivalent transistor circuits characterizing the modes of operation of the devices. The most common small-signal equivalent circuit diagram of the P-MOSFET is shown in Figure 4(a) [9]. 
International Journal of Computational Science, Information Technology and Control Engineering

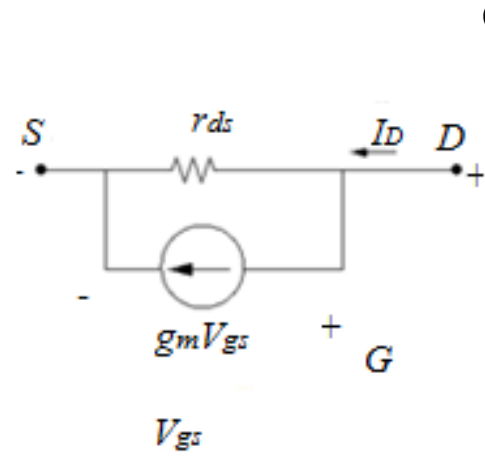

(a)
(IJCSITCE) Vol.3, No.3, July 2016

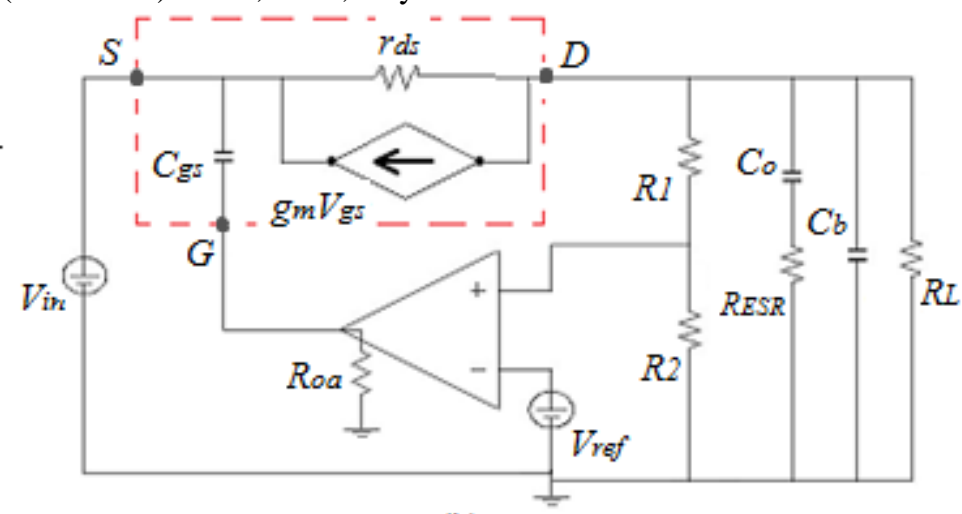

(b)

Figure 4. Small signal model of the PMOS pass element

In this model, the drain-source circuit is represented by an equivalent current source, which is valid in the linear region, where the output characteristics of the transistor are horizontal. For a description of this model, one needs to know the transconductance $\mathrm{g}_{\mathrm{m}}$ and the drain-source onstate resistance $r_{d s}$. Typical values of $g_{m}$ are $0.5-5 \mathrm{~mA} / \mathrm{V}$, and for $r_{d s}$, values range between a few miliohms to several tens of ohms. In practice, $g_{m}$ can be found from the slope of the output characteristics curve of the transistor. The transfer function of the series pass MOSFET is $G_{m o s}(s)=g_{m} r_{d s}$ and the error amplifier has a constant gain $G_{e a}(s)=k_{o}$. Therefore, the transfer function of the MOSFET pass element in series with the error amplifier is

$$
T_{e m}(s)=G_{e a}(s) G_{m o s}(s)=k_{o} g_{m} r_{d s}
$$

The described model contains only resistive components, and does not account for the inertial properties of the transistor. This resistive model is usually valid at low frequencies of input signals. When considering the small signal operation of the FET in the high-frequency region, it is necessary to use a FET model comprising energy storage elements (Figure 4(b)). As follows from the structure of the field effect transistor, there is capacitive coupling between the gate and the channel. The value of capacitance is small - of the order of a few picofarads. In this case, the regulator will have the form shown in Figure 4(b) with a forward transfer function

$$
G_{e a}(s) G_{m o s}(s)=\frac{k_{o} g_{m} r_{d s}}{1+s R_{o a} C_{g s}}
$$

The overall closed loop transfer function with regulator output filter and load is given by

$$
T(s)=\frac{G_{e a}(s) G_{m o s}(s) G_{f l}(s)}{1+\beta G_{e a}(s) G_{m o s}(s) G_{f l}(s)}
$$

Where $\beta=\frac{R_{2}}{R_{1}+R_{2}}$ is the feedback factor, and $G_{f l}(s)$ is the transfer function of filter and load, given by: 
International Journal of Computational Science, Information Technology and Control Engineering (IJCSITCE) Vol.3, No.3, July 2016

$G_{f l}(s)=\frac{Z_{o}(s)}{r_{d s}+Z_{o}(s)}$

where $Z_{o}(s)$ is the output impedance.

From Figure 4(b) $Z_{o}(s)=\frac{R_{L}\left(s R_{E S R} C_{o}+1\right)}{s^{2} R_{L} C_{b} C_{o} R_{E S R}+s\left[\left(R_{L}+R_{E S R}\right) C_{o}+R_{L} C_{b}\right]+1}$, and so $G_{f l}(s)$ can be written as

$G_{f l}(s)=\frac{R_{L}\left(s C_{o} R_{E S R}+1\right)}{\left(R_{L}+r_{d s}\right)+s\left[\left(R_{L}+R_{E S R}\right) C_{o}+R_{L}\left(r_{d s} C_{b}+C_{o} R_{E S R}\right)\right]+s^{2} R_{L} R_{E S R} C_{o} C_{b} r_{d s}}$

The overall forward transfer function $T_{f}(s)$ of the power regulator system is thus

$T_{f}(s)=\frac{g_{m} k_{o} r_{d s} R_{L}\left(s C_{o} R_{E S R}+1\right)}{\left(R_{L}+r_{d s}\right)+s\left[\left(R_{L}+R_{E S R}\right) C_{o}+R_{L}\left(r_{d s} C_{b}+C_{o} R_{E S R}\right)\right]+s^{2} R_{L} R_{E S R} C_{o} C_{b} r_{d s}} \times \frac{1}{1+s R_{o a} C_{g s}}$

And the close-loop transfer function can be written as

$T(s)=\frac{R_{L} R_{T}\left(s C_{o} R_{E S R}+1\right)}{\alpha+s \beta+s^{2} \chi+s^{3} \delta}$

where the coefficients $\alpha, \beta, \chi$ and $R_{T}$ have the following meanings:

$$
\begin{aligned}
& R_{T}=R_{1}+R_{2} \\
& \alpha=R_{1}\left(R_{L}+r_{d s}\right)+R_{2} r_{d s}\left(g_{m} k_{o} R_{L}+1\right)+R_{2} R_{L} \\
& \beta=R_{T}\left[C_{o}\left(R_{L}+R_{E S R}\right)+C_{g s} R_{o a}\left(R_{L}+r_{d s}\right)+R_{1}\left(C_{o} R_{E S R}+C_{b} r_{d s}\right)+g_{m} k_{o} r_{d s} R_{L} C_{o} R_{E S R}\right] \\
& \chi=R_{T}\left[C_{o} C_{g s} R_{o a}\left(R_{L}+R_{c}\right)+C_{g s} R_{o a} R_{1}\left(C_{o} R_{E S R}+C_{b} r_{d s}\right)+C_{b} C_{o} R_{E S R} R_{L} r_{d s}\right] \\
& \delta=R_{T}\left[C_{b} C_{o} C_{g s} R_{o a} R_{E S R} R_{L} r_{d s}\right]
\end{aligned}
$$

\section{AnAlysis AND TUNING OF THE CONTROL SCHEME}

\subsection{Setting Practical Component Values for Analysis}

To validate the developed model and design a practical PID controller we obtain numerical values for the coefficients of the model to be used in the controller design. Practical component values, given by datasheets [10-11] and other guides [12-15] are provided in Table 1. 
International Journal of Computational Science, Information Technology and Control Engineering

(IJCSITCE) Vol.3, No.3, July 2016

Table 1. Summary of the regulator parameter specifications

\begin{tabular}{|l|l|l|l|l|l|l|l|l|l|l|l|}
\hline Parameter & $R_{L}$ & $C_{b}$ & $C_{o}$ & $C_{g s}$ & $R_{o a}$ & $R_{E S R}$ & $g_{m}$ & $R_{1}$ & $R_{2}$ & $R_{d s}$ & $k_{o}$ \\
\hline Value & 2 & 0.1 & 10 & 300 & 300 & 5 & 0.5 & 2 & 0.5 & 6 & 26.021 \\
\hline Unit & $k \Omega$ & $\mu F$ & $\mu F$ & $p F$ & $k \Omega$ & $\Omega$ & $m A / V$ & $k \Omega$ & $k \Omega$ & $m \Omega$ & $d B$ \\
& & & & & & & & & & & \\
\hline
\end{tabular}

From these values the transfer function (9) can then be written explicitly as:

$$
T_{f}(s)=\frac{3.0 \times 10^{16}\left(5.0 \times 10^{-6} s+1\right)}{9 s^{3}+7.701750 \times 10^{9} s^{2}+2.572250 \times 10^{16} s+5.000015 \times 10^{20}}
$$

\subsection{Determining PID Settings}

From the mathematical model of the LDO regulator, the PID controller parameters can be determined. In this study, the PID controller parameters are determined using the Ziegler-Nichols ultimate cycle method [16]. To calculate the controller parameters using the ultimate cycle method requires only two coefficients: the critical proportional gain $K_{c}$, and the associated period of oscillations $T_{c}$. Knowing the coefficients $K_{c}$ and $T_{c}$ of the controlled system, it is easy to calculate the proportional gain $K_{p}$, integral and derivative time constants, $T_{i}$ and $T_{d}$. The formulas for calculating PID parameters $K_{p}, T_{i}$ and $T_{d}$, first proposed by Ziegler and Nichols [35], are given in Table 2.

Table 2. PID parameter settings

\begin{tabular}{|l|c|c|c|}
\hline PID Parameter & $K_{p}$ & $T_{i}$ & $T_{d}$ \\
\hline Definition & $0.6 K_{c}$ & $T_{c} / 2$ & $T_{c} / 8$ \\
\hline
\end{tabular}

To find $K_{c}$ all control modes, except proportional, are turned off. $K_{p}$ is first set to a low value and continually increased gradually while observing the system response to a step change in the set point for the different values of gain $K_{p}$. The gain value at which the system begins to develop sustained oscillations with constant amplitude is the critical gain $K_{c}$. The period of oscillation at this maximum gain limit is the critical period $T_{c}$. This procedure can be carried out analytically by setting the integration time constant to infinity while the time constant of differentiation is set to zero or the minimum possible value. The proportional gain $K_{c}$, and the associated period of oscillations $T_{c}$ are obtained on the basis of the limits of stability of the closed loop system with proportional-only controller. Switching off the integral and differential components of the controller results in a closed loop transfer function with the characteristic equation:

$$
9.0 s^{3}+7.70175 \times 10^{9} s^{2}+\left(25722.50+0.03 K_{c}\right) \times 10^{12} s+\left(5.00015+0.60 K_{c}\right) \times 10^{20}=0
$$

The numerical value for the critical coefficient $K_{c}$ is determined using the Routh-Hurwitz criterion. The Routh array for the given characteristic polynomial is 
International Journal of Computational Science, Information Technology and Control Engineering (IJCSITCE) Vol.3, No.3, July 2016

$$
\left[\begin{array}{ccc}
9.0 & \left(2572250+3.0 K_{c}\right) \times 10^{10} & 0 \\
7.70175 \times 10^{9} & \left(500.015+30.0 K_{c}\right) \times 10^{18} & 0 \\
\left(2572192-4.01139 K_{c}\right) \times 10^{10} & 0 & 0 \\
\left(500.015+30.0 K_{c}\right) \times 10^{18} & 0 & 0
\end{array}\right]
$$

At the boundary of stability, $\left(2572192-4.01139 K_{c}\right) \times 10^{10}=0$ and so $K_{c}=6.412 \times 10^{5}$. The frequency of oscillations is obtained from the auxiliary equation

$$
7.70175 \times 10^{9} s^{2}+\left(500.015+30.0 K_{c}\right) \times 10^{18}=0
$$

Thus, $\omega=7.068 \times 10^{7} \mathrm{rad} / \mathrm{s}$ and the critical period of oscillations $T_{c}=\frac{2 \pi}{\omega}=8.890 \times 10^{-8} \mathrm{~s}$.

From table 1, all PID parameters can then be calculated:

$$
\begin{aligned}
k_{p} & =0.6 K_{c}=3.847 \times 10^{5} \\
\tau_{i} & =\frac{T_{c}}{2}=4.445 \times 10^{-8} \mathrm{~s} \\
\tau_{d} & =\frac{T_{c}}{8}=1.111 \times 10^{-8} \mathrm{~s}
\end{aligned}
$$

Therefore, the PID controller transfer function given by equation 2 has the numerical representation:

$$
k_{p}\left(1+\frac{1}{\tau_{i} s}+\tau_{d} s\right)=3.847 \times 10^{5}+\frac{8.971 \times 10^{10}}{s}+4.274 \times 10^{-3} s
$$

\section{TEST AND RESULTS}

To test the performance of the proposed system, the response of the closed loop system with the calculated PID settings was simulated. The graph of Figure 5 shows the settling time in response to a step input to be $0.0213 \mathrm{~ms}$, the overshoot is approximately $4.3 \%$ and the rise time is $3.03 \mu \mathrm{s}$. It is important to keep in mind that settings obtained by analytical calculations do not always give optimal controller settings, as analytical results are based on a highly simplified model of the object and are, therefore, approximations. Therefore, after the calculation of the controller parameters, it is desirable to make adjustments. Adjustments are made on the basis of rules that are used for PID tuning [17-19]. It is also noteworthy that the application of these rules is only possible after predetermining the controller parameters experimentally or by formulas. Setting up the controller without an initial approximate calculation of the coefficients may be unsuccessful, as the rules are valid only in the vicinity of the optimal controller settings. 
International Journal of Computational Science, Information Technology and Control Engineering (IJCSITCE) Vol.3, No.3, July 2016

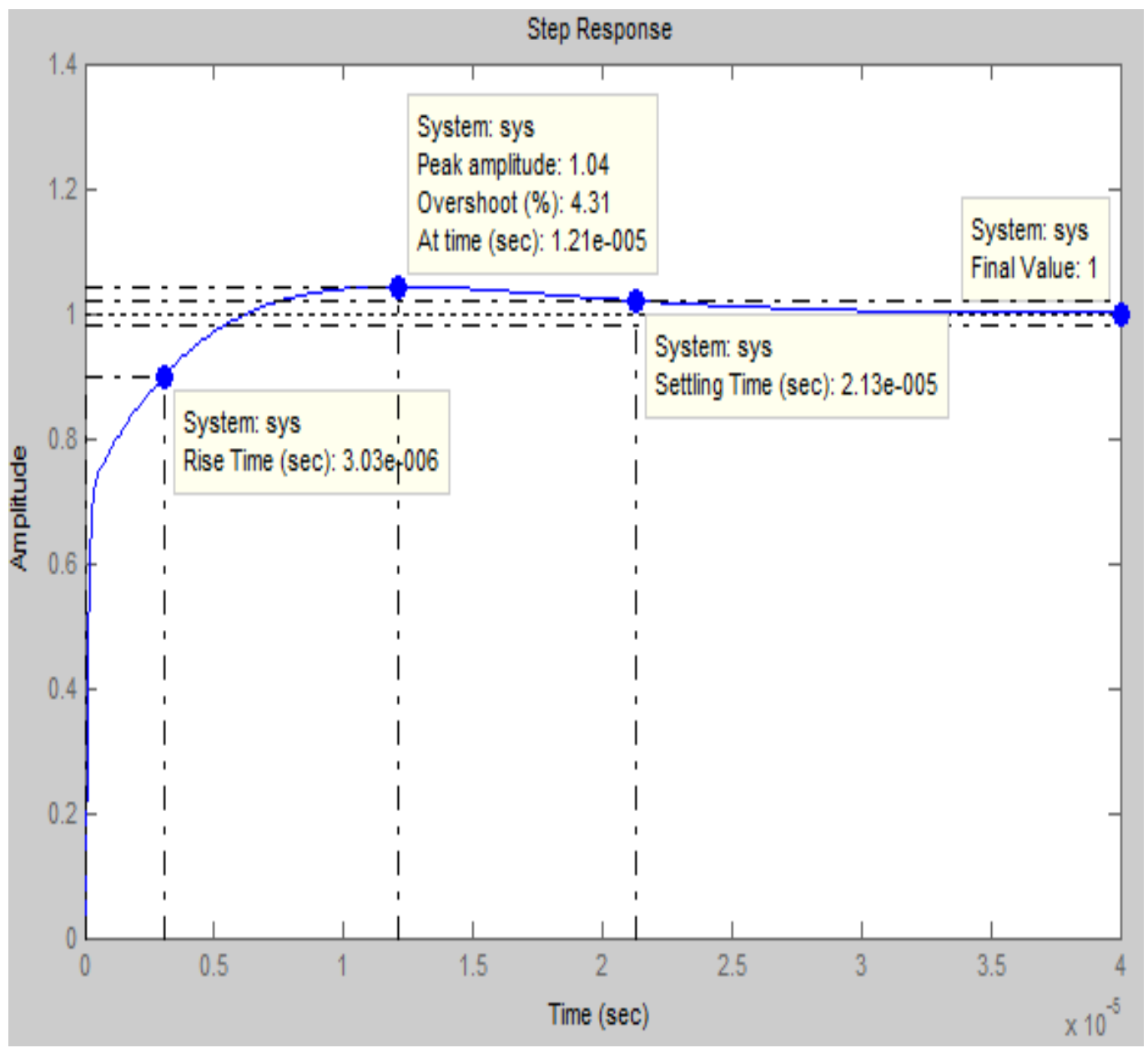

Figure 5. Transient response of the LDO regulator with calculated PID parameters

Using manual adjustment procedures it was possible to improve the performance of the system. The transient response of the closed loop regulator when the coefficients $k_{p}=9.85 \times 10^{5}$, $\tau_{i}=1.152 \times 10^{-7} \mathrm{~s}$ and $\tau_{d}=9.645 \times 10^{-6} \mathrm{~s}$ has the following characteristics:

- $\quad$ Settling time - 265 nano seconds

- $\quad$ Rise time - 114 nano seconds

- $\quad$ Peak overshoot- $0 \%$

Figure 6 shows the response obtained after the adjustment of the PID parameters. As can be seen from the curve, the speed of the response, and the peak overshoot have drastically improved without adversely affecting steady state error. 
International Journal of Computational Science, Information Technology and Control Engineering (IJCSITCE) Vol.3, No.3, July 2016

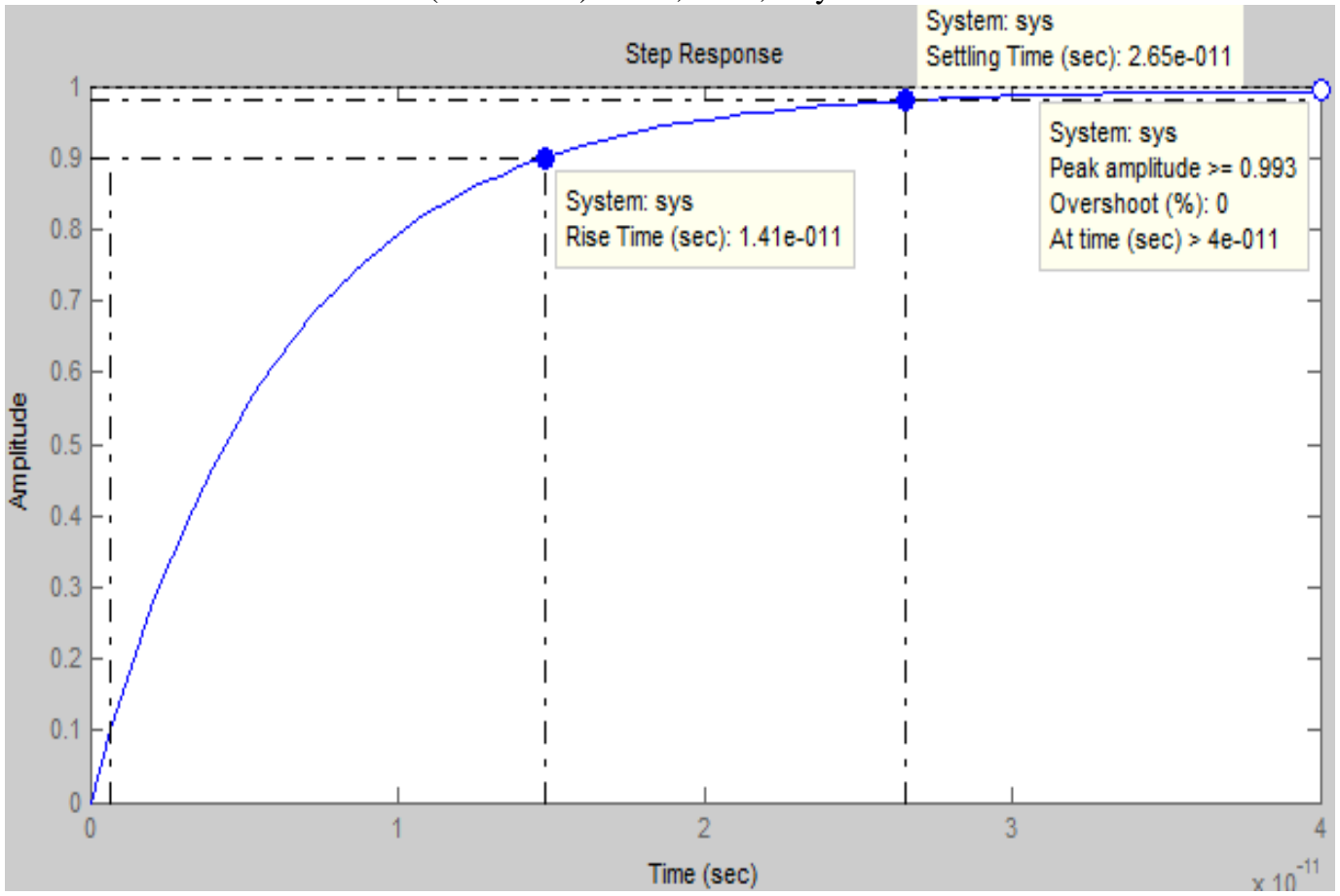

Figure 6. Transient response of the LDO regulator with adjustment of PID settings

Finally, a bode plot of the system with the calculated PID parameter settings is shown in Figure 7. From the bode plot, the system has phase margin of $86.7^{\circ}$ and a gain margin of infinity. This means that it is sufficiently stable.

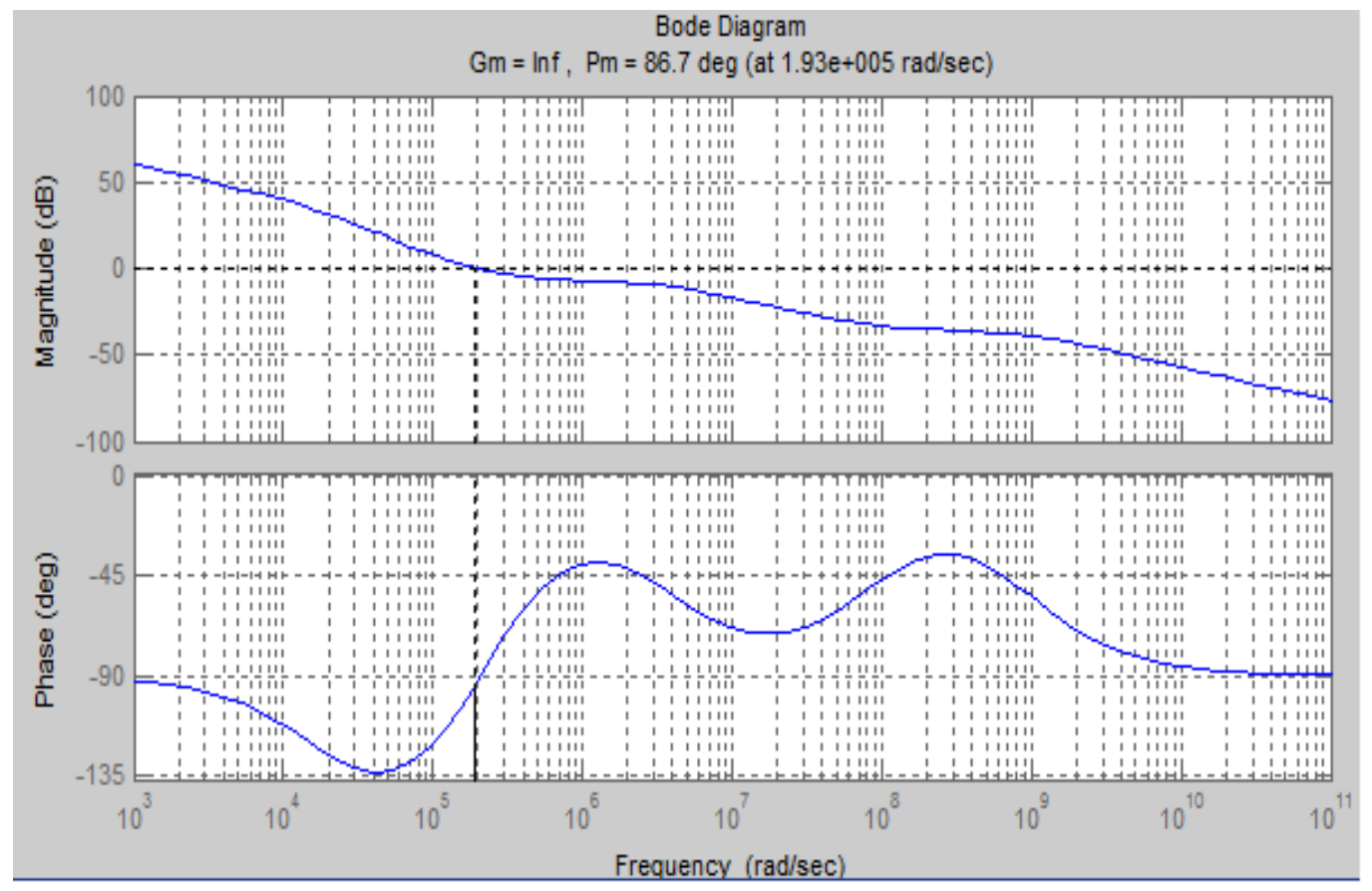

Figure 7 Bode plot of the LDO regulator with calculated parameters 
International Journal of Computational Science, Information Technology and Control Engineering (IJCSITCE) Vol.3, No.3, July 2016

\section{CONCLUSIONS}

When adjusting controller settings for high performance electrical processes, manual tuning based on prescribed rules can take a lot of time and resources. Therefore, in this study an analytical method was used to design a PID based control system for a linear regulator. Theoretical analysis of the linear regulator based on a series pass FET transistor amplifier provided a simple relation that accurately described the operation of the regulator. The mathematical model obtained was used to determine the parameters of the control system. The stated method for determining controller settings greatly reduces design time and cost. Analysis of the proposed design shows that the PID controller based control system provides good regulation performance and stable operation for the regulator with the specified control parameters. Thus, better dynamic characteristics of LDO regulator and its cost effective realization are achievable using PID control techniques, as this allows the use of analytical methods to determine optimum controller settings.

\section{REFERENCES}

[1] K. Lee, "Advanced Control Schemes for Voltage Regulators," Ph.D. Dissertation, Virginia Tech, Blacksburg, VA, 2008.

[2] M A. Aziz, A. Mahfouz and D. M. Khorshied, "Simplified approaches for controlling dc-dc power converters," International Journal of Engineering Science and Technology, vol. 4, no. 2, , 2012, pp. 828-840.

[3] V. S. C. Raviraj and P. C. Sen, "Comparative Study of Proportional-Integral, Sliding Mode, and Fuzzy Logic Controllers for Power Converters," IEEE Transactions on Industry Applications, vol. 33, no. 2, 1997.

[4] S. S. Chong, "Design of high performance low-dropout regulators for on-chip applications," Ph.D dissertation, Sch. of Electrical and Electronic Eng., Nanyang Tech. Univ., 2014.

[5] Z.Li, L. He, and C. Chen, "A novel low-dropout regulator with large load current and high stability," in Advanced Research and Technology in Industry Applications, IEEE Workshop on, 2014, pp. $1122-1126$.

[6] Y. I Kim and S. S. Lee, "A capacitorless LDO regulator with fast feedback technique and lowquiescent current error amplifier," Circuits and Systems II: Express Briefs, IEEE Transactions on, vol. 60, no. 6, 2013, pp. 326-330.

[7] R. Vilanova and A Visioli, PID control in the third millennium, London: Springer, 2012.

[8] K. Rishi and N. Goyal, "Study and Analysis of Small Signal Parameters, Slew Rate and Power Dissipation of Bipolar Junction Transistor and Complementary MOS Amplifiers With and Without Negative Feedback Using T-Spice," International Journal of Science and Research, vol. 3 no.8, 2014.

[9] A.K. Maimi and V. Aggrawal, Electronic Devices and Circuits, Wiley Publications, African Reprint, New Delhi, 2010.

[10] "Understanding power MOSFET datasheet parameters," Application Note, NXP, rev. 2-16, August 2012. [Online]. Available: www.nxp.com/ documents/application_note/ AN11158.pdf. [Accessed: Dec. 2, 2015].

[11] A. Huang, "Infineon OptiMOS ${ }^{\mathrm{TM}}$ Power MOSFET Datasheet Explanation," Application Note, AN 2012-03, vol. 1.1, Mar. 2012. [Online]. Available: www.infineon.com/.../Infineon++Application+Note+-+OptiMOS+Power... [Accessed: Oct. 14, 2015].

[12] B. S. Lee, "Understanding the stable range of equivalent series resistance of an LDO regulator," Analog Applications, 1999.

[13] "Linear Regulators: Theory of Operation and Compensation," Application Report, Texas Instruments Inc., lliterature number SNVA020B, May, 2000-Revised May, 2013. [Online]. Available: www.ti.com/lit/an/snva020b/snva020b.pdf. [Accessed: Jul. 20, 2015].

[14] E. Rogers, "Stability analysis of low-dropout linear regulators with a PMOS pass element," Analog Applications, 1999. 
International Journal of Computational Science, Information Technology and Control Engineering

(IJCSITCE) Vol.3, No.3, July 2016

[15] C. Simpson, "Linear Regulators: Theory of Operation and Compensation, " National Semiconductor Application Note, AN-1188, May 2000. [Online]. Available:

www.farnell.com/datasheets/1359239.pdf. [Accessed: Nov. 4, 2015].

[16] J.G. Ziegler and N.B. Nichols, "Optimum settings for automatic controllers," trans. ASME, vol. 64, no. $11,1942$.

[17] A. O'Dwyer, Handbook of PI and PID controller tuning rules, vol. 57, London: Imperial College Press, 2009.

[18] O. Lequin, M. Gevers, M. Mossberg, E. Bosmans and L. Triest, "Iterative feedback tuning of PID parameters Comparison with classical tuning rules," Control Eng. Pract., vol. 11, no. 9, 2003, pp.1023 -1033.

[19] M. G. Lin, S. Lakshminarayanan and G. P.Rangaiah, "A comparative study of recent/popular PID tuning rules for stable, first-order plus dead time, single-input single-output processes," Industrial \& Engineering Chemistry Research, vol. 47, no. 2, 2008, pp.344-368. 